\title{
Delivering effective genetic services for patients and families affected by cleft lip and/or palate
}

\author{
Nicola Marie Stock $\mathbb{D}^{1} \cdot$ Rhona MacLeod ${ }^{2} \cdot$ Jill Clayton-Smith ${ }^{2}$
}

Received: 6 August 2018 / Revised: 26 November 2018 / Accepted: 4 December 2018 / Published online: 25 January 2019

(c) European Society of Human Genetics 2019

\begin{abstract}
Genetic services for individuals affected by cleft lip and/or palate (CL/P) and their families are an important aspect of clinical care; yet debate exists as to how this service should be offered. This study explored the utility, acceptability, and delivery of genetic services from the perspectives of cleft-specialist clinicians, genetic counsellors, and affected families. Analysis of data collected from three focus groups and eleven individual interviews identified two overarching themes "Referring patients and families to genetic services" and "The role of a genetic specialist in the context of CL/P". The first examines the common reasons for referral to the genetics service, how best to judge the timing of a referral, and the optimal approach to the delivery of sensitive genetic information. The second theme discusses the role of the genetic specialist in the context of cleft care, including the optimal management of affected individuals and their families, and the delivery of basic genetics training and support for health professionals working in other disciplines. A model for the effective delivery of genetic services in CL/P is subsequently proposed. Coordination and financial implications of the proposed model ultimately require further consideration and evaluation to determine its effectiveness.
\end{abstract}

\section{Introduction}

Cleft lip and/or palate (CL/P) is a common congenital anomaly occurring in approximately one in every 500-700 live births around the world [1]. Approximately $50 \%$ of all clefts occur as isolated malformations and are likely to be multifactorial in origin, caused by a complex interplay between numerous susceptibility genes in combination with environmental factors [2]. Multifactorial clefts have a relatively low recurrence risk, and therefore routine genetic tests do not usually reveal any abnormalities. The remaining $50 \%$ of clefts, the majority of which are clefts of the palate only, occur in association with additional malformations, growth problems and/or developmental delay [3]. These syndromic forms may be

$\triangle$ Nicola Marie Stock

Nicola2.Stock@uwe.ac.uk

1 Centre for Appearance Research, University of the West of England, Coldharbour Lane, Bristol BS16 1QY, UK

2 Manchester Centre for Genomic Medicine, Central Manchester University Hospitals, Saint Mary's Hospital, Oxford Road, Manchester M13 9WL, UK due to chromosomal abnormalities, changes in single genes and/or certain environmental factors, such as exposure to particular medications or alcohol during pregnancy [3]. Management of syndromic clefts can be extremely complex, and in addition to routine cleft care, this involves screening for associated medical complications and regular surveillance of the child's growth and development. Genetic testing can be helpful in obtaining an accurate diagnosis, guiding the management of the patient's care, and assessing recurrence risk for parents and other family members.

In the United Kingdom (UK), genetic counselling was added to the $\mathrm{CL} / \mathrm{P}$ treatment pathway later than other aspects of care. In 1996, the minimum standards for the management of CL/P published by the Royal College of Surgeons [4] called for the provision of genetic counselling for all patients and their families. Past research has also emphasised the importance of access to genetic counselling for the CL/P population [5, 6]. Yet, due largely to the complex aetiology of clefting, the needs of affected individuals and their family members in terms of genetic input vary widely. While some may benefit considerably from genetic investigation and advice, others may not gain as much from a consultation, and thus a referral to the genetics service may be of lower priority 
than the interventions offered by other members of the $\mathrm{CL} / \mathrm{P}$ team. Further, some people may prefer not to engage in genetic services at all [6]. A survey carried out by the UK Clinical Genetics Group (see www.cgs.org.uk) demonstrated the debate among clinical geneticists as to which $\mathrm{CL} / \mathrm{P}$ patients require a formal genetic opinion and at what point(s) during the care pathway this should be offered. A further consideration relates to the integration of genetic testing into routine care (mainstreaming) and the training and support of health professionals to deliver some aspects of genetic counselling for the more straightforward testing scenarios.

Genetic investigation can also have significant psychological ramifications, not just for patients, but also for partners, the wider family and subsequent generations. Previous studies within CL/P and the wider health field have highlighted the importance of managing perceptions and expectations of genetic counselling [7, 8], as well as the need for health professionals to possess a high level of knowledge and sensitivity when delivering genetic information [6,9]. With the advent of new technologies such as chromosome microarray analysis and whole-exome and -genome sequencing, the information to be imparted to families has become more complex, and significant skill is required, not just to communicate newer types of information to families but also to obtain consent from them to genetic testing beforehand [10]. Affected individuals and their family members also require an appropriate follow-up after attending a genetic consultation and support to facilitate the coping process $[8,11]$. Genetic referral, consultation and follow-up therefore need to be approached and managed carefully.

It is clear that although many could benefit significantly from genetic services and therefore access to this service is of vital importance, a standardised approach may not be suitable for all. To date, little consideration has been given to the role of genetic services in the context of CL/P. The aim of the present study was therefore to explore the utility, acceptability, and delivery of genetic counselling within cleft care, from the perspectives of UK-based cleft-specialist clinicians, genetic counsellors, and affected young adults and parents.

\section{Materials and methods}

\section{Design}

The present study involved the use of semi-structured focus groups and telephone interviews to elucidate the views of cleft-specialist clinicians, clinical geneticists, genetic counsellors, young adults born with $\mathrm{CL} / \mathrm{P}$, and parents of children born with CL/P.

\section{Procedure}

Ethical approval for the study was obtained through the Faculty Ethics Committee at (university). Cleft-specialist clinicians and clinical geneticists/genetic counsellors were invited to participate via an advertisement distributed via email. Parents and young adults were identified through the regional Specialist Health Visitor or by a member of the $\mathrm{CL} / \mathrm{P}$ teams. All participants received information about the study, including ethical considerations, such as their right to withdraw, and were given opportunities to ask any question. Participants were asked to provide their informed consent prior to data collection.

One focus group was conducted with cleft-specialist clinicians and one with clinical geneticists/genetic counsellors. Focus groups were held in a private room in a hospital setting, with each group lasting for $60-90 \mathrm{~min}$. Interview topics for clinical geneticists/genetic counsellors included existing protocols for referral and follow-up, benefits and challenges of genetic services in the context of CL/P, and optimal service delivery. Additional interview topics for cleft-specialist clinicians included clinicians' current knowledge of aetiology and recurrence rates of $\mathrm{CL} / \mathrm{P}$.

Parents of children with CL/P and young adults with CL/ $\mathrm{P}$ were interviewed individually over the telephone. Telephone interviews were conducted at a mutually convenient time and lasted $60 \mathrm{~min}$ on average. Interview topics included knowledge of genetics and the role of a geneticist in the context of CL/P, prior experiences of referrals and genetic testing, and optimal service delivery.

Data collection was carried out by the second and third authors who are trained in qualitative methodology. All focus groups and interviews were audio-recorded with the participants' permission and transcribed verbatim with patient-identifiable information removed.

\section{Participants}

Eight senior cleft-specialist clinicians from two regional cleft teams participated in this study. Participants represented a number of disciplines involved in cleft care, including plastic/reconstructive surgery, speech and language therapy, nursing, orthodontics, and psychology. Five senior clinical geneticists/genetic counsellors working across two regions also participated.

Sampling of the five parents and six young adults was purposeful to ensure the representation of syndromic and non-syndromic cases, as well as a range of cleft phenotypes. Parents were all mothers, aged 28-37 years (mean age = 32.8 years). Their children (three females, two males) had been born with cleft lip and palate $(n=3)$ or cleft palate only $(n=2)$. Two of their children had been diagnosed with 
a syndrome. Young adults (five females, one male) were aged $16-30$ years (mean age $=23.2$ years). Five had been born with a cleft lip and palate, and one with cleft palate only.

\section{Data analysis}

An attenuated form of grounded theory was performed [12, 13] using open and axial coding, memoing and the constantcomparative method, supported by ATLASti software. Transcripts were read several times to familiarise the authors with the data, and any word or phrase that initially stood out was highlighted. Initial codes were then identified, and similarities and differences between the codes were examined, in order to identify how codes clustered together. Analysis was performed separately by the first and second authors, and themes were cross-checked to ensure full agreement.

\section{Results}

Data analysis identified two main themes and a number of relevant subthemes across all three participant groups. Findings are presented below in accordance with each theme and in Table 1. The findings of this study have also informed the design of a model that offers suggestions for the optimal delivery of genetic services in the context of CL/P. This model is illustrated in Fig. 1.

\section{Referring patients and families to genetic services}

\section{Reasons for referral}

Cleft-specialist clinicians and clinical geneticists/genetic counsellors described the main circumstances in which they would anticipate a referral to genetic services. This included families with a known history of CL/P, parents wanting to have another baby, and families who actively requested a consultation with a genetic specialist. A referral would commonly be considered if the child presented with 'dysmorphic features' or another major anomaly, and/or if a developmental delay was suspected. Participants also suggested that young adults with $\mathrm{CL} / \mathrm{P}$, as well as patients' partners and siblings, may require information regarding recurrence risk. It was raised by some cleft-specialist clinicians that 'all' patients and families should be referred to genetic services in order to normalise this as a routine part of care. However, other cleft-specialist clinicians and clinical geneticists/genetic counsellors disagreed on the basis that it could be frustrating for families with no clear genetic implications and unnecessarily anxiety-provoking. Overall, the opinion of participants was for the genetics service to remain external to, but closely linked with, the specialist cleft team.

\section{Timing of referral}

All participants agreed that the timing of the referral is crucial and that the appropriate time to approach the subject of genetics with each family/patient is likely to vary considerably. Participants commented on the need to consider the psychological impact of a referral to the genetics service on the patient, the parents and the wider family. Cleftspecialist clinicians and clinical geneticists/genetic counsellors identified a number of additional circumstances in which genetic referrals may not be appropriate or may require further consideration. This included situations in which the two parents disagreed on whether to pursue genetic counselling and questions surrounding the identity of the biological father. Nonetheless, some cleft-specialist clinicians and clinical geneticists/genetic counsellors warned against missing opportunities to conduct a genetic assessment, and suggested that blood tests should be done as a standard at the time of surgery. Finally, cleft specialist clinicians suggested that 'sowing seeds' over time in relation to referral to the genetics service could be beneficial for some families.

\section{Delivery of information}

All participants indicated that many families/patients did not possess a good understanding of genetics. Concurrently, cleft-specialist clinicians discussed the importance of managing patients' and families' preconceptions and expectations of genetic services. All participant groups identified non-specialist health professionals, such as general practitioners (GPs), health visitors and paediatricians as a possible initial source of basic information for individuals with $\mathrm{CL} / \mathrm{P}$ and their families. However, some cleft-specialist clinicians raised concerns over information misgiving or insensitivity to the potential psychological impact of information delivery. Consequently, all participants believed that information about genetics should initially be delivered by a member of the CL/P team where possible. A number of participants felt that having a clinical geneticist available at the multidisciplinary team clinic could be a potential opportunity to raise issues relating to aetiology and recurrence. However, when discussing recurrence risks with affected young adults and their siblings, opinion was divided as to whether it was best to be seen alone or with the family present.

Some participants, particularly young adults, felt that supplementary information regarding the genetics of $\mathrm{CL} / \mathrm{P}$ and what a consultation with the genetics service might involve should be provided prior to the consultation. 


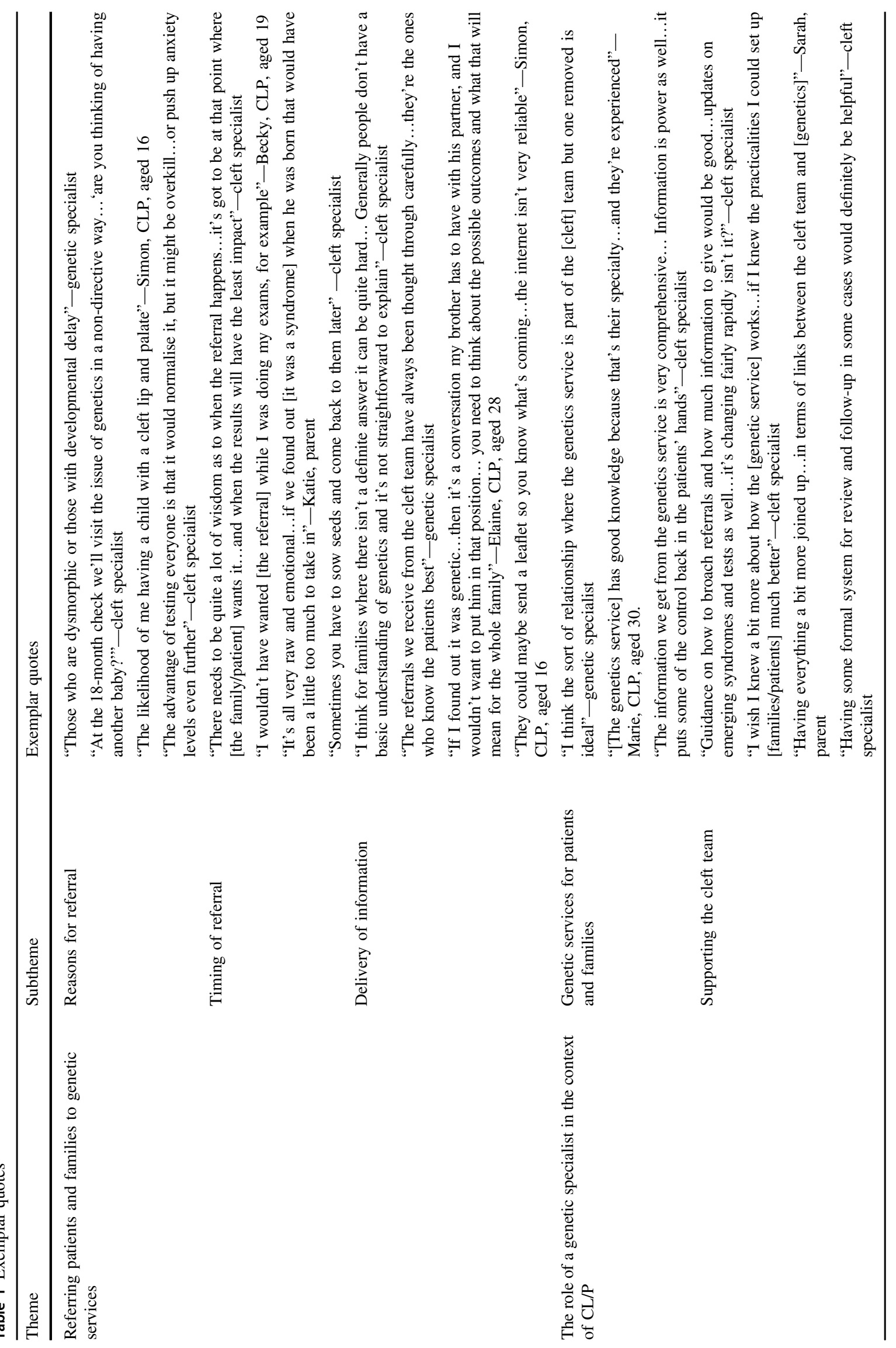


Fig. 1 A proposed model for the effective delivery of genetic services in the context of cleft care

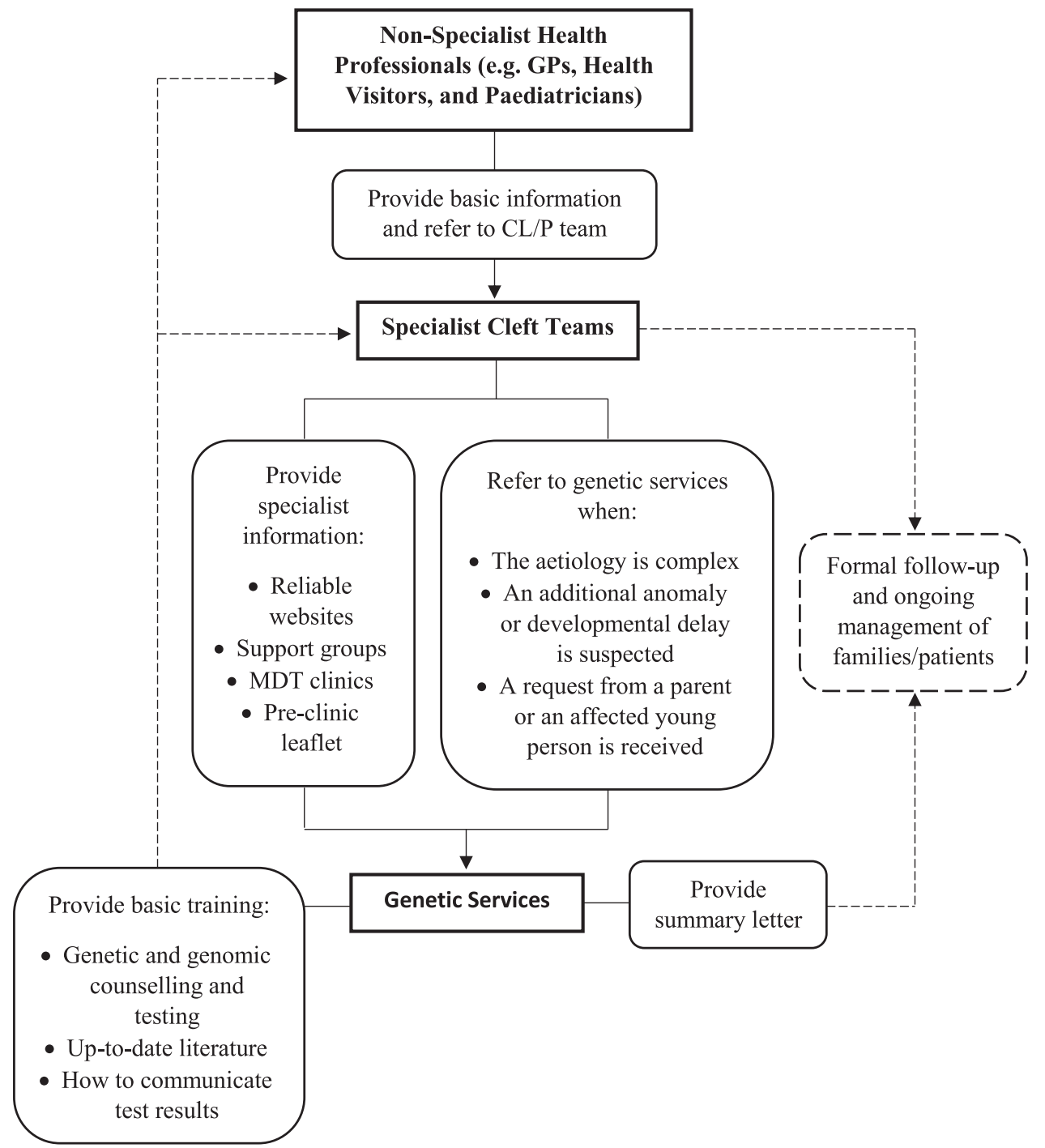

Participants suggested that receiving a generic or conditionspecific leaflet could be one way of achieving this. Parents and young adults in particular also commented on the pros and cons of information obtainable from the internet, with a predominant concern that information was not wellmonitored.

\section{The role of a genetic specialist in the context of $\mathrm{CL} / \mathrm{P}$}

\section{Genetic services for patients and families}

The role of a genetic specialist was seen predominantly as educating families/patients in basic genetics, providing diagnoses where relevant and conducting risk assessments. Cleft-specialist clinicians and clinical geneticists/genetic counsellors generally agreed that the genetics service should be peripheral to the $\mathrm{CL} / \mathrm{P}$ team, rather than being intrinsic within the CL/P service. Parents and young adults valued the genetics service highly, particularly in relation to the opportunity to access reliable information from an 'expert'. Equally, cleft-specialist clinicians indicated that the information the genetics service provided to both the cleft team and patients and families was of a high standard.

\section{Supporting the cleft team}

Cleft-specialist clinicians indicated strongly that they would appreciate guidance on how best to refer families and patients to the genetics service. They also stated that up-todate information on the different genetic diagnoses would be appreciated, particularly in light of how rapidly the genetics field is moving. Cleft specialist clinicians commented that their knowledge of how their local genetic service operates and how it is structured could also be improved in order to support the families and patients more effectively. They mentioned that advice from the genetics service with regard to how to follow families/patients up after a diagnosis was also important, as well as links to 
relevant support organisations. Ongoing communication and feedback between the CL/P team and the genetics service was discussed by all participant groups, with cleftspecialist clinicians commenting that having a more formal follow-up process would be of benefit to them and their patients.

\section{Discussion}

This study has provided a unique insight into the utility, acceptability, and delivery of genetic counselling from the perspectives of cleft-specialist clinicians, genetic counsellors, and affected young adults and parents. Overall, participants viewed the genetic services to be an important part of the CL/P treatment pathway. However, given that genetic information is complex and potentially impactful, information, referral, and follow-up should be based on individual need. Participants provided guidance as to how to best address these inherent challenges, and a model was subsequently proposed (Fig. 1). Suggestions on how to best structure the genetics service in the context of CL/P are discussed in further detail below.

\section{Implementing an optimal model of referral}

In 1996, guidance published by the Royal College of Surgeons [4] called for the provision of genetic counselling for all patients and their families affected by CL/P. In contrast, the findings from the present study imply there are many situations in which a referral to genetic services may not be appropriate, or in which the families may gain little from a genetic consultation. Participants identified patients and families with a complex aetiology and children with other major anomalies and/or suspected developmental delay to represent those who would most benefit from a genetic consultation. Additionally, it was thought that those families or patients who actively request a consultation should be referred to the genetics service. In comparison, it was felt that parents from affected families wanting to have another baby, young adults affected by CL/P thinking about starting a family, and patients' partners and siblings may benefit from receiving a more basic level of genetic information, which could be effectively delivered by the cleft team. While there is a clear need for access to genetic services in the case of CL/P $[5,6]$, a more targeted allocation of the limited resources available is necessary in order to ensure that all families and patients are given priority access to those interventions that are most appropriate in the context of their individual needs.

Further, the timing at which a referral to the genetics service is offered may present challenges due to the personal circumstances of the patient or family member.
Adjustment to $\mathrm{CL} / \mathrm{P}$ and its treatment involves a highly complex interplay of social, cultural, physical and psychological factors, and is known to fluctuate over time and in accordance with various life stages and events [14]. Thus, the timing of any intervention must be handled sensitively. All participants felt that a member of the CL/P team, particularly the specialist cleft nurse, would be most familiar with families and patients and would therefore be best placed to make this judgement, with support from the surrounding team. Nonetheless, some points of opportunity common to all families and patients were identified. These included the idea of collecting DNA from the child at the time of surgery to prevent further blood tests later on, raising the issue of further family planning at the 18-month follow-up appointment, and approaching the young adults with the topic of heritability prior to their 'discharge' from the service. While some degree of judgement from the CL/P team will likely always be required, more general guidance on the timing of referrals may help to standardise the service.

\section{Managing patient expectations}

Although genetic testing has moved on considerably in recent years, it is clear from reports obtained through this study and others [6] that public understanding of genetics within the CL/P community remains relatively low. In addition, previous studies examining the effectiveness of genetic counselling more broadly have reported that families and patients place a high value on the certainty of information [7]. This is potentially problematic given that the aetiology and recurrence risk of CL/P is particularly complex [2, 3]. Thus, preconceptions and expectations of what the genetics service can realistically deliver for families and patients need to be managed prior to any consultation $[8,15]$.

\section{Provision of generic and tailored patient information}

Concurrently, the findings from the present study suggest that the combination of general information immediately following the receipt of a genetics referral, along with the more tailored information obtained during the consultation itself, would be a favourable model. Previous studies have discussed the idea of providing a leaflet containing basic information about genetics, along with advice on how to prepare for the consultation and what questions to ask $[16,17]$. Leaflets were also promoted as a means of communicating this information and reducing anxiety in the current study, and it was indicated that such leaflets may already be in circulation in other areas of health care. Further evaluation of this method of information delivery in the 
context of CL/P may be warranted [7]. The delivery of genetic information via the internet was also discussed in the present study. Although the control of online material is ultimately limited, monitored and reliable guidance from a validated source is likely to reduce any fears and support the management of preconceptions $[17,18]$, and should thus be made a priority.

\section{Increasing knowledge of genetics among health professionals}

Another significant topic discussed by the participants was the importance of an appropriate level of genetic knowledge among members of the CL/P team, as well as among nonspecialist health professionals, such as GPs, health visitors and paediatricians. In the current study, genetic specialists identified one of their roles to be the training of CL/P team members in basic genetic knowledge, a responsibility that is reflected in a number of European reports [19]. Equally, cleft-specialist clinicians commented they would welcome guidance on how to best approach the referrals, a better understanding of how the genetics service works, up to date information regarding diagnoses and the current best practice and advice regarding reliable support groups. Future work could support the development of a training module for the professionals involved in cleft care that could be offered as an e-learning module or as a component of designated courses, for example, the UK MSc in Genomic Medicine. Concerns regarding the accuracy and sensitivity with which information is delivered by non-specialists have been raised in a number of previous CL/P studies [17, 20, 21] and remains an important issue for cleft care. This approach could therefore be extended to non-specialist health professionals, such as midwives, GPs, and health visitors, who are also likely to have regular contact with the families and patients affected by CL/P.

\section{Collaborative management and follow-up}

Finally, the positioning of the genetics service within cleft care was debated at length among the different groups of participants. The proposal that genetic specialists should be integral to the CL/P team and should attend multidisciplinary team clinics regularly was favoured by a few participants, with the motivation of 'normalising' genetic referrals and reducing the overall number of hospital appointments. Nonetheless, the majority of participants believed that while the two services should be closely linked, the genetics service should remain peripheral to the everyday delivery of cleft care carried out by the CL/P team. This model has been used effectively within other areas of health care and was recommended following a review of genetic services in Europe [22]. Elsewhere, a number of calls have been made with regard to the mainstreaming of genetic services in order to make genetic testing more accessible. The majority of examples to date originate from the field of oncology [23-25], where mainstreaming initiatives have facilitated the co-location of genetic and oncology delivery while separating the clinical responsibility for (cascade) genetic testing to a specialist genetics service [25]. The potential move toward the mainstreaming of genetic testing in the context of cleft care could be an important future consideration. Cleft-specialist clinicians also discussed the value of working closely with the genetics service after the consultation with the family or patient has taken place [8]. As one aspect of this, the quality and importance of the summary letters that the genetics service provides following a consultation was emphasised. It has been suggested in a wider CL/P literature that families and patients may struggle to assimilate information at times of acute or ongoing stress [26], and therefore a summary letter that can be taken home may help to facilitate understanding to develop over time. Implementing a more formal follow-up procedure for families and patients to ensure ongoing and collaborative management of their care pathway may be a positive step forward in developing CL/P services.

\section{Study limitations}

Methodological limitations of the current study must be acknowledged. While a total sample of 24 participants is generally considered adequate for a small-scale qualitative study [27], data were derived from three different participant groups using two different data collection methods. While combining different methods in this manner is not uncommon [28], data collected in a private one-to-one dialogue may differ from those collected in a group setting. Only 11 individual interviews were conducted. Although a variety of different phenotypes were purposefully sought to ensure broad representation, data were subsequently indicative of a spectrum of experiences. Equally, only two focus groups were conducted. As a result, the data collected may not have reached full saturation and/or may not be entirely applicable outside of the two regions. Nonetheless, common themes were observed across the data collected from all participants, and the findings contribute multiple and valuable perspectives on a subject that has not previously received significant attention.

\section{Conclusions}

This original qualitative study explored the utility, acceptability, and delivery of genetic counselling within the context of cleft care, from the perspectives of cleft-specialist 
clinicians, genetic counsellors, and affected young adults and parents. The findings have been used to suggest a model for the effective delivery of genetic services for patients affected by CL/P and their families. Further work is needed to expand upon the current findings and to evaluate the proposed model to determine its effectiveness and acceptability.

Acknowledgements The authors would like to acknowledge all the participants for their contribution and all those who supported the study. The authors have no funding to report.

\section{Compliance with ethical standards}

Conflict of interest The authors declare that they have no conflict of interest.

Publisher's note: Springer Nature remains neutral with regard to jurisdictional claims in published maps and institutional affiliations.

\section{References}

1. World Health Organization. Oral health: Factsheet 318; 2016. http://www.who.int/mediacentre/factsheets/fs318/en.

2. Mossey PA, Little J, Munger RG, Dixon MJ, Shaw WC. Cleft lip and palate. Lancet. 2009;374:1773-85.

3. Dixon MJ, Marazita ML, Beaty TH, Murray JC. Cleft lip and palate: understanding genetic and environmental influences. Nat Rev. Gen. 2011;12:167-78.

4. Shaw WC, Williams AC, Sandy JR, Devlin HB. Minimum standards for the management of cleft lip and palate: efforts to close the audit loop. Ann R Coll Surg Engl. 1996;78:110-4.

5. Monlleó IL, Gil-da-Silva-Lopes VL. Brazil's craniofacial project: genetic evaluation and counselling in the reference network for craniofacial treatment. Cleft Palate Craniofac J. 2006;43:577-9.

6. Stock NM, Rumsey N. Starting a family: the experience of parents with cleft lip and/or palate. Cleft Palate Craniofac J. 2015a;52:425-36.

7. MacLeod R, Craufurd D, Booth K. Patients' perceptions of what makes genetic counselling effective: an interpretative phenomenological analysis. J Health Psychol. 2002;7:145-56.

8. Broder $\mathrm{H}$, Trier WC. Effectiveness of genetic counselling for families with craniofacial anomalies. Cleft Palate J. 1985;22:157-62.

9. von der Lippe C, Frich JC, Harris A, Solbrække KN. Experiences of being heterozygous for Fabry Disease: a qualitative study. J Genet Couns. 2016;25:1085-92.

10. Ormond KE. From genetic counseling to "genomic counseling". Mole Genet Genomic Med. 2013;1:189-93.
11. Li KC, Birch PH, Garrett BM, MacPhee M, Adam S, Friedman JM. Parents' perspectives on supporting their decision making in genome-wide sequencing. J Nurs Scholarsh. 2016;48:265-75.

12. Glaser BG, Strauss AL. The discovery of grounded theory: strategies for qualitative research. New York: Aldine de Gruyter; 1967.

13. McAllister M. Grounded theory in genetic counselling research. J Gen Couns. 2001;10:233-50.

14. Rumsey N, Stock NM. Living with a cleft: psychological challenges, support and intervention. In: Berkowitz S, editor. Cleft Lip and Palate: Diagnosis and Management. $3^{\text {rd }}$ ed. Berlin Heidelberg: Springer-Verlag; 2013.

15. Michie S, Marteau T, Bobrow M. Genetic counselling: the psychological impact of meeting patients' expectations. J Med Gen. 1997;34:237-41.

16. Hallowell N, Murton F, Statham H, Green JM, Richards MP. Women's need for information before attending genetic counselling for familial breast or ovarian cancer: a questionnaire, interview and observational study. BMJ. 1997;314:281-3.

17. Knapke SC, Bender P, Prows C, Schultz JR, Saal HM. Parental perspectives of children born with cleft lip and/or palate: a qualitative assessment of suggestions for healthcare improvements and interventions. Cleft Palate Craniofac J. 2010;47:143-50.

18. Lee K, Hoti K, Hughes JD, Emmerton LM. Consumer use of "Dr Google": a survey on health information-seeking behaviours and navigational needs. J Med Int Res. 2015;29:e288.

19. Godard B, Kääriäinen $H$, Kristoffersson $U$, Tranebjaerg L, Coviello D, Aymé S. Provision of genetic services in Europe: current practices and issues. Eur J Hum Genet. 2003;11:S13-S48.

20. Nelson PA, Kirk SA. Parents' perspectives of cleft lip and/or palate services: a qualitative interview. Cleft Palate Craniofac J. 2013;50:275-85.

21. Stock NM, Rumsey N. Parenting a child with a cleft: the father's perspective. Cleft Palate Craniofac J. 2015b;52:31-43.

22. Harris R, Reid M. Medical genetic services in 31 countries: an overview. Eur J Hum Genet. 1997;5:3-21.

23. Katz SJ, Kurian AW, Morrow M. Treatment decision making and genetic testing for breast cancer: mainstreaming mutations. JAMA. 2015;314:997-8.

24. George A, Riddell D, Seal S, Talukdar S, Mahamdallie S, Ruark E et al. Implementing rapid, robust, cost-effective, patient-centred, routine genetic testing in ovarian cancer patients. Sci Rep. 2016;13:29506.

25. Kentwell M, Dow E, Antill Y, Wrede CD, McNally O, Higgs E et al. Mainstreaming cancer genetics: a model integrating germline BRCA testing into routine ovarian cancer clinics. Gynecol Oncol. 2017;145:130-6.

26. Collett BR, Speltz ML. Social-emotional development of infants and young children with orofacial clefts. Infants \& Young Children. 2006;19:262-91.

27. Morse JM. Determining sample size. Qual Health Res. 2000;10:3-5.

28. Creswell JW, Plano-Clark VL. Designing and Conducting Mixed Methods Research. 2nd ed. Thousand Oaks, CA: Sage; 2011. 